\title{
New Roles for Old Glue: Astrocyte Function in Synaptic Plasticity and Neurological Disorders
}

\author{
Syed M. Q. Hussaini ${ }^{1}$, Mi Hyeon Jang ${ }^{2,3}$ \\ ${ }^{1}$ Department of Internal Medicine, Duke University Medical Center, Durham, NC, USA \\ ${ }^{2}$ Department of Neurologic Surgery, Mayo Clinic, Rochester, MN, USA \\ ${ }^{3}$ Department of Biochemistry and Molecular Biology, Mayo Clinic, Rochester, MN, USA
}

Previously believed to solely play a supportive role in the central nervous system, astrocytes are now considered active players in normal brain function. Evidence in recent decades extends their contributions beyond the classically held brain glue role; it's now known that astrocytes act as a unique excitable component with functions extending into local network modulation, synaptic plasticity, and memory formation, and postinjury repair. In this review article, we highlight our growing understanding of astrocyte function and physiology, the increasing role of gliotransmitters in neuron-glia communication, and the role of astrocytes in modulating synaptic plasticity and cognitive function. Owing to the duality of both beneficial and deleterious roles attributed to astrocytes, we also discuss the implications of this new knowledge as it applies to neurological disorders including Alzheimer disease, epilepsy, and schizophrenia.

Keywords: Astrocyte; Central nervous system; Synaptic plasticity; Neurological disorders

- Fund/Grant Support: This work was supported by NIA (R01AG058560), Regenerative Medicine Minnesota, Mayo Clinic Center for Regenerative Medicine, and Department of Neurologic Surgery at Mayo Clinic.

- Conflict of Interest: No potential conflict of interest relevant to this article was reported.

\section{INTRODUCTION}

While neurons are undoubtedly the classic stars of neuroscience research, recent research forces us to expand the spotlight to the star-shaped astrocytic glia. For over a century, astrocytes have been considered both stagehand and scaffold within the central nervous system (CNS). In the recognized supportive role of astrocytes, their known functions ranged from providing structure, literally making up the "glue" that bound the neuronal elements together, to vasomodulation and maintaining the blood-brain barrier. The past 30 years have pushed our understanding into new directions and roles for astrocytes such as modulation of synaptic transmission, long-term potentiation (LTP), and postinjury repair in the nervous system.

In this article, we review recent evidence expanding on the known roles of astrocytes in the CNS. We provide an overview of astrocytes, their incredible heterogeneity, and how they are physiologically stimulated to transmit information and influence synaptic transmission. This is followed with a description of gliotransmitters and their specific roles in mediating or

Corresponding author: Mi Hyeon Jang (iD http://orcid.org/0000-0001-6274-3588 Department of Neurologic Surgery, Mayo Clinic College of Medicine, 200 First Street SW, Rochester, MN 55905, USA

E-mail: jang.mihyeon@mayo.edu / Tel: +1-507-284-1219 / Fax: +1-507-284-3383

Co-corresponding author: Syed M.Q Hussaini

(i) http://orcid.org/0000-0002-8006-5343

Department of Internal Medicine, Duke University Medical Center, 2301 Erwin

Rd, Durham, North Carolina 27705, USA

E-mail: smh87@duke.edu / Tel: +1-919-603-8861

Submitted: September 17, 2018 / Accepted after revision: October 10, 2018 
modulating long-term potentiation. Finally, we review recent evidence relevant to astrocyte function in CNS pathologies such as Alzheimer disease, depression, epilepsy, and schizophrenia. Where possible, relevant clinical and in vivo work has been utilized and where helpful, other reviews have been referenced to provide the reader with understanding on topics beyond the scope of this work.

\section{ASTROCYTES AS DIVERSE NEURAL CIRCUIT ELEMENTS}

A pervading thought in neuroscience, and more specifically, glia research, is that neuroglia outnumber neurons 10:1 [1]. Recent evidence, however, shows that this number may be vastly overestimated. Using isotropic fractionation of human brain samples combined with NeuN nuclei labelling, research now establishes the ratio of neuronal to non-neuronal cells is closer to 1:1 [2]. This ratio is in line with other studies [3]. Interestingly, these studies also found that this ratio varies throughout the brain. In cerebral cortex, there is an increase in glia relative to neurons whereas in the cerebellum it is the opposite [2,3].

While the exact reasons for these shifts in glial populations are unknown, it has been suggested that increased neuronal size and coinciding metabolic demand explains the need for increased glial support [2,3]. Indeed, cortical regions show increased glia: neuron ratios across animal species, suggesting that glia may be of evolutionary importance. Similarly, using a combination of glial fibrillary acidic protein (GFAP) and S100 calcium binding protein B (S100B), markers primarily expressed in astrocytes, at least nine different astrocyte populations may be identified that are phenotypically diverse, but region specific to the extent that they may be used to delineate different anatomical regions in the brain [4]. The unique morphology and excitability of astrocytes allows them to taken on several structural roles in the CNS that include maintenance of the blood brain barrier, ion homeostasis, and regulation of neuron-neuron communication [5].

This heterogeneity gives a level of versatility to the astrocyte that allows it to have profound effects on the surrounding neuronal network. A novel study recently published supports the potential evolutionary role of astrocytes in promoting cognitive ability. Using cultured human glial progenitor cells engrafted into neonatal mice, Han et al [6]. demonstrated that these glial progenitors differentiate to become astrocytes and show enhanced function. These glia differentiated into mature astro- cytes, integrated into the existing host astroglial network, exhibited faster propagation of $\mathrm{Ca}^{2+}$ signaling, and promoted LTP. Furthermore, these human glia chimeric mice demonstrated increased cognitive ability as demonstrated by improved performance in the Barnes maze, object-location tasks, alongside contextual and tone fear conditioning tasks [6]. These studies support the notion that astrocytes are heterogeneous elements contributing to cognitive function, either through homeostatic maintenance or other mechanisms.

Anatomically, astroglial are stereotypically identified by their star-shaped morphology. However, as mentioned above, they also exhibit substantial heterogeneity that may explain their expansive roles within the nervous system [7]. While astrocytes may be classified based on morphology, this can often be difficult due to their wide variation in appearance. Thus, the most widely used methods of identifying astrocytes are the molecular marker, GFAP and S100B [8,9]. Both markers have been shown to be sensitive to the major astrocyte types, protoplasmic and fibrous [10]. Protoplasmic astrocytes are commonly found in grey matter and are characterized by their fine, almost cloudlike, processes enveloping neuronal synapses. Fibrous astrocytes, found in white matter, differ in that they exhibit thin and defined processes which are unbranched and whose end-feet meet neuronal nodes of Ranvier. While the aforementioned markers and morphologic phenotypes are useful for broad characterization of astrocytes, other classes of astroglia exist.

\section{ASTROCYTES AND COMMUNICATION}

\section{Calcium Waves and Astrocyte Excitability}

Astrocytes had long been considered passive members of the CNS without electrical activity. It wasn't until the 1990s when new techniques in $\mathrm{Ca}^{2+}$ imaging revealed them as excitable, albeit in ways different from the neuron. The earliest studies demonstrated how cultured hippocampal astrocytes responded to glutamate with increases in intracellular calcium [11], or that mechanical stimulation of a single astrocyte in a primary glial culture could increase intracellular calcium concentration [12]. In both instances, specific increases in intracellular calcium were propagated to cells in the surrounding cultures providing us with some of the earliest evidence of communication between astrocytes. Gap junctions between local astrocytes in addition to extracellular adenosine triphosphate (ATP) link this activity to the surrounding glia and onward in an electrically coupled syncytium $[13,14]$. Relatively slow $\mathrm{Ca}^{2+}$ waves, as com- 
pared to the neuronal action potential, are now recognized as hallmark features of astrocytes.

Astrocytes undergo both neuron-dependent and spontaneous excitation [15]. The neuron-dependent excitation of astrocytes is a well-documented way in which synaptic neurotransmitters directly or indirectly through the connected astrocyte syncytium lead to astrocyte excitation. Astrocytes respond to a wide range of neurotransmitters including glutamate, gamma-aminobutyric acid (GABA), acetylcholine, ATP, nitric oxide, and brain-derived neurotrophic factor (BDNF) [15,16]. Further, there is evidence that astrocytes are able to discriminate synapses belonging to different axon pathways. In rat hippocampal slices, Perea and Araque [17] showed that astrocytes not only respond differently to glutamate and acetylcholine, but also to glutamate released from the Schaeffer collaterals compared to glutamate from the alveus terminals. Beyond neuron-dependent excitation, astrocytes are capable of generating spontaneous intracellular calcium oscillations that trigger astrocytic glutamate release causing $\mathrm{N}$-methyl$D$-aspartate (NMDA)-receptor dependent neuronal activation [18]. Similarly, hippocampal astrocytes in situ exhibit calcium oscillations that occur independent of neuronal activity or metabotropic glutamate and purinergic receptors and are mediated by inositol-phosphate-3 receptor activation [19].

$\mathrm{Ca}^{2+}$ signaling regulation varies by the anatomical region that the astrocyte is found in. A recent study shows that astrocytes along the hippocampal mossy fiber pathway respond to repeated mossy fiber action potentials, are activated by neurotransmitters glutamate and GABA, and cover a large brain region indicating unsuitability in mediating individual synapses [20]. Importantly, observed spontaneous astrocyte $\mathrm{Ca}^{2+}$ signaling was independent of this activity and its associated neurotransmitters. In contrast, previous study of hippocampal CA1 and dentate gyrus astrocytes show a high sensitivity towards spontaneous activity by glutamate and single action potentials at a local level [21,22]. Such differences in astrocyte $\mathrm{Ca}^{2+}$ response sensitivity, effect size, and overall stimulus suggest differential neuronal population effects. Interestingly, the anatomical placement of these astrocytes along this circuit hints at their function. As part of the trisynaptic circuit, hippocampal input follows the perforant path where entorhinal cortical inputs synapse within the dentate gyrus, which then via mossy fibers is relayed to CA3, which is ultimately relayed to CA1 via schaffer collaterals. Such astrocyte heterogeneity along this circuit suggests differential function based on factors such as synapse number, source of inputs and outputs, in addition to variation in plasticity within these anatomical regions. The extent and significance of this astrocyte variation has yet to be systematically explored.

Together, growing evidence on the excitation of astrocytes establishes them as integral elements in the information-processing pathways of the CNS capable of contributing to excitation of both glial and neuronal elements. It's important to note that $\mathrm{Ca}^{2+}$ signaling and its role in astrocyte function is rapidly changing and its effects may be too fine to adequately determine without reassessment [23].

\section{Gliotransmission}

$\mathrm{Ca}^{2+}$ astrocyte activation is required for the release of astrocyte gliotransmitters [21,24]. Volterra and Meldolesi have previously suggested a precise classification criterion for gliotransmitters. This definition includes synthesis and/or storage in astrocytes, a regulated release stimulated by physiological stimuli, activation of rapid responses in neighboring cells, and finally a role of the gliotransmitters in physiological processes [15]. Even within this growing list of gliotransmitters, molecules released by the astrocyte may not all hold the same breadth of function or effect; some including D-serine may act as strong mediators of synaptic plasticity and LTP, while others such as ATP and tumor necrosis factor (TNF)- $\alpha$ may act as modulators and regulate plasticity in more subtle ways [25].

By releasing gliotransmitters to communicate over short distances with neighboring neurons, astrocytes actively contribute to functions spanning synapse development/transmission to controlling blood vessel flow. There is now a growing list of molecules secreted by astrocytes in response to stimulation. These include glutamate, ATP, adenosine, D-serine, prostaglandins and TNF- $\alpha$ among many others that are secreted from the astrocyte and act at specific receptors with specific outcomes. Glutamate is perhaps one of the most well-known gliotransmitter released by astrocytes. It acts at the alpha-amino-3-hydroxy5-methyl-4-isoxazolepropionic acid (AMPA) and NMDA-receptors, and metabotropic glutamate receptors (mGluRs) and contributes to LTP by increasing excitatory postsynaptic potentials and upregulating AMPA receptors [5]. As discussed later in this review, other secreted gliotransmitters such as D-serine, ATP and TNF- $\alpha$ significantly contribute to LTP [25].

Mechanistically, gliotransmitter release from astrocytes is attributed to both exocytosis-mediated and more recently nonexocytosis pathways. Glutamate release by astrocytes occurs classically through exocytosis in a $\mathrm{Ca}^{2+}$-dependent manner pre- 
viously thought specific only to synapses. This may occur through a synaptic-like vesicular (SLMV) compartment that contains crucial elements required for glutamate exocytosis, including the vesicular glutamate transporter, vesicular SNARE protein, and cellubrevin $[15,26]$. Interestingly, the gliotransmitter D-serine that regulates NMDA-receptor mediated LTP may be coreleased with these vesicles or SLMVs carrying glutamate [27]. As more evidence accumulates, it is possible for gliotransmitters to be released from $\mathrm{Ca}^{2+}$-independent nonexocytotic mechanisms. These include hemichannels such as the $\mathrm{Cx} 43$ prevalent in astrocytes, volume-regulated anion channels or VRACs, purinergic channels such as $\mathrm{P}_{2} \mathrm{X}_{7}$ or the cysteine-glutamate exchanger [28].

\section{Astrocytes as Neuronal Mediators: LTP}

With expanding knowledge on astrocyte signaling and gliotransmission as it pertains to the tripartite synapse, research has expanded on the neuronal functional outcomes of this phenomenon on LTP. LTP is the process by which varied biological processes may be reinforced. This popularly includes memory formation but may also include formation of addiction behavior with drugs. Instead of considering the presynaptic and postsynaptic neurons in isolation, the astrocyte has become increasingly recognized as part of a tripartite model where it acts as an essential 3rd element actively involved in how a signal may be conducted at the neuronal synapse.

LTP mechanisms have been extensively studied in the past two decades. The most well studied mechanisms are those involving NMDA-dependent glutamate signaling and a cAMPdependent presynaptic form of plasticity that we will elaborate on here. For LTP induction, both the presynaptic and postsynaptic neurons must be depolarized, following which there much be high frequency stimulation of the NMDA-type glutamate receptors that results in a large amount of calcium influx into the post-synaptic neuron [29]. This influx leads to activation of calmodulin kinase II, phosphorylation of AMPA receptors, and their exocytosis to the postsynaptic membrane $[29,30]$. The requirement of AMPA receptors for NMDA-dependent LTP induction was first understood from experiments involving activation of postsynaptically silent synapses in the hippocampus $[31,32]$. It is now a well evidenced in the literature as the mechanism of LTP induction [29].

The corollary to LTP is long-term depression (LTD) that as the name suggests is a reduction in activity-dependent reduction in synaptic strength. It has gained increasing attention in recent years with its functional importance studied in monocular deprivation studies that lead to blindness in the unused eye. Of the several mechanisms that have been posited, NMDA-receptor dependent LTD is a leading one. Experimentally, LTD may be induced through low-frequency stimulation $(1-3 \mathrm{~Hz}$ for 5-15 minutes) of the presynaptic neurons [29]. Repetitive stimulation such as this results in a prolonged increase in calcium in the postsynaptic neuron due to repeated stimulation of the NMDA receptors [29]. The calcium influx selectively activates phosphatases that cause endocytosis of, and reduce the AMPA receptors at the synaptic cleft.

Glutamate is the primary excitatory neurotransmitter in the brain and also the primary neurotransmitter for LTP and LTD induction, acting through the ionotropic NMDA and AMPAtype glutamate receptors. Astrocytes play significant roles in regulating LTP induction. They are the primary recyclers of glutamate and sequester up to $90 \%$ of the neurotransmitter at the synaptic cleft [33-35]. In fact, early experiments that quantified glutamate-uptake by astrocytes (astrocyte transporter currents) played a pivotal role in our understanding of LTP induction, namely that LTP induction is not related to further glutamate release, but rather an intracellular response involving AMPARs as described above [36,37]. Astrocytes also release glutamate through a calcium-regulated mechanism that allow for astrocyte-neuron communication [26,38,39]. Triggers include neuron-dependent excitation of astrocytes through neurotransmitters including synaptic glutamate itself that can act through astrocytic metabotropic glutamate receptors [15]. In the hippocampus, glutamate release from astrocytes also synchronizes neuronal activity and control synaptic strength [40].

\section{ASTROCYTES AND NEUROLOGICAL DISORDERS}

As we have increasingly understood a more complex and widespread role of astrocytes in the CNS, studies have also demonstrated the involvement of astrocytes in neurological disorders. Astrocytes can be both causative, and protective, in pathological states of neurodegeneration. Below we discuss recent evidence in our understanding of how the astrocyte contributes to observed neurological disorder pathology.

\section{Alzheimer Disease}

Alzheimer disease $(\mathrm{AD})$ is characterized at the cellular level by widespread neurofibrillary tangles composed of hyperphosphorylated tau protein and neuritic plaques composed of the 
amyloid beta proteins. While the exact etiology of $\mathrm{AD}$ is unknown, the prevailing hypothesis that aggregation of fibrillogenic amyloid beta over time leads to cellular, and thus cognitive, dysfunction is well supported by genetic and molecular study [41]. Additionally, AD related degeneration coincides with astrocyte reactive gliosis [42].

Mouse models of AD show evidence of altered intracellular calcium signaling within the astrocytes. For instance, in the APP/PS1 mouse model of AD producing excess amyloid, timelapse calcium imaging shows significant increase in spontaneous astrocyte activity related to the age-specific development of plaques (3.5 months vs. 6 months) [43]. Both the frequency and amplitude of these transient calcium currents were higher in the APP/PS1 mice, coordinated over long distances and independent of local neuronal activity. Furthermore, application of amyloid-beta to mixed culture of hippocampal astrocytes of neurons causes selective induction of calcium currents in astrocytes compared to neurons [44]. While there is calcium-dependent glutathione depletion in both neurons and astrocytes, the $50 \%$ observed death in neurons could be secondary to the neuronal-dependence on astrocytes for antioxidant support.

Besides such pathological changes observed in AD brains that are associated with reactive astrogliosis, one other avenue of active research has been in understanding the observed cognitive impairments, namely, the slow-onset memory loss. GABA signaling has provided a potential explanation for the cognitive deficits. In APP/PS1 mice, the application of bicuculine, a $\mathrm{GABA}_{\mathrm{A}}$ antagonist, to hippocampal slices of both adult APP/PS1 and old nontransgenic mice increased the observed LTP, while application of picrotoxin, another $\mathrm{GABA}_{\mathrm{A}}$ antagonist could rescue the memory deficits in APP/PS1 mice [45]. Further, in both the cerebellum and hippocampus, tonic inhibition is directly correlated with astrocytic GABA which is released through the Bestrophin-1 channel $[46,47]$. Indeed, it was recently shown in the APP/PS1 model that reactive astrocytes may use monoamine oxidase- $\mathrm{B}$ to produce and release GABA from the Best1 channels [48]. The increased GABA was directly related to impaired presynaptic probability, spike probability, synaptic plasticity, and learning and memory. Both putrescine and amyloid-beta caused Maob-mediated GABA production and release from Best1 channels, while the resulting deficits could be fully rescued by using Maob inhibitors such as selegeline or targeting the Best1 channels. The study is the clearest evidence thus far of astrocytes playing a key role in mediating memory loss in the hippocampus.

\section{Major Depression}

Depression is a common disorder that is characterized by anhedonia, loss of interest, psychomotor retardation, and suicidality. It is a significant cause of disability worldwide with $16.2 \%$ lifetime and 6.6\% 12-month prevalence in the United States population [49]. Several studies in humans have recorded pathological glial loss in the brains of major depressive disorder (MDD) patients. These include glial reduction in the subgenual prefrontal cortex, anterior cingulate gyrus, and prefrontal cortex [50-52]. In the hippocampus, several magnetic resonance imaging studies reveal a decreased volume [53]. Interestingly, while another study found depth of hippocampal slices from post-mortem brains of MDD patients, at the cellular level there was a $30 \%$ increase in glial density across hippocampal pyramidal subfields and the granular cell layer. Compared to other regions in the brain with glial loss, in the hippocampus however there was a unique reduction in neuropil that consists of glial cells, their processes, dendrites and axonal processes that may explain the volume loss [54]. These studies show that astrocytes are pathological hallmarks of MDD.

Several studies have attempted to delineate how astrocytes may mediate MDD behaviors. Previous research establishes that L-alpha-aminoadipic acid (L-AA) selectively ablates astrocytes. Targeting the prefrontal cortex, studies show that L-AA astrocyte ablation leads to anhedonia in sucrose preference test, anxiety in novelty suppressed feeding test, and overall increased helplessness in forced swimming and two-way active avoidance tests in vivo [52,55]. Additionally, the role of astrocytes in mediating depressive-live behavior may be directly related to its release of the gliotransmitter ATP. Mice that are susceptible to chronic social defeat, a model that mimics depression, may have lower levels of ATP in the prefrontal cortex and the hippocampus. In their study, Cao et al. [56] showed that administration of ATP produces rapid antidepressant effects while blocking ATP release in mice lacking IP3 receptor type 2 or by inhibiting vesicular gliotransmission produces depressive-like behavior. Furthermore, using a transgenic Gq GPCR astrocyte mouse model to selectively activate astrocytes through $\mathrm{Ca}^{2+}$ signaling and ATP release, Cao et al. [56] were able to rescue some depressive behaviors in mouse models of depression. Supporting the role of astrocyte purinergic signaling, at least one study looking at the ventral PFC in patients with suicide showed altered expression of genes involving ATP biosynthesis in addition to GABA transmission [57]. 


\section{Epilepsy}

Different mechanisms have been put forth to describe the role of astrocytes in epilepsy. Human studies of patients with temporal lobe epilepsy have shown evidence of reactive astrogliosis. One manner in which astrocytes may mediate epilepsy includes dysfunction of the glutamate-glutamine cycle. The increase in GFAP and vimentin that occur with reactive astrogliosis has been associated with a concurrent decrease in glutamine synthetase, an enzyme that converts glutamate to glutamine which is a precursor to the neurotransmitter GABA. It has been hypothesized that this results in a lack of inhibitory synaptic transmission in the surrounding neurons. In their study, Ortinski et al. [58] showed how adeno-associated virus induction of reactive astrogliosis in mouse CA1 pyramidal neurons reduced the inhibitory input on the surrounding neurons. Reduced inhibitory postsynaptic current was observed that could be increased with exogenous administration of glutamine.

Astrocytes may also result in epileptiform activity through their effects on adenosine levels. This comprises the adenosine kinase (ADK) hypothesis of epileptogenesis. Adenosine plays neuroprotective roles in seizure regulation with its levels typically elevated following a seizure. It may mediate seizure arrest and postseizure refractoriness. Adenosine is converted to adenosine monophosphate via the enzyme ADK which is predominantly found in the astrocytes. Reactive astrogliosis causes an increased amount of available ADK that can potentially act on adenosine and reduce its levels leading to seizure aggravation [59]. Finally, increasing evidence is demonstrating that calcium oscillations in, and glutamate release from astrocytes could also lead to epileptiform discharges. Cultured astrocytes from human epileptic foci show increased spontaneous calcium-oscillation frequency $[60,61]$. Inducing cortical epileptiform activity may increase calcium-oscillations in astrocytes that can be suppressed though the use of anticonvulsants. Further, astrocytes may also cause excitations in nearby neurons that resemble interictal paroxysmal depolarizations. Other studies have posited that glutamate release from astrocytes instead of being necessary to cause epileptiform discharges could play more of a modulatory role $[62,63]$. Other studies have shown calciumdependent glutamate release from astrocytes may also contribute to neuronal death seen in status epilepticus [64].

\section{Schizophrenia}

Astrocyte role in the pathogenesis of schizophrenia comes from recent studies involving the gliotransmitter $\mathrm{D}$-serine and
NMDA neurotransmission that bear on the glutamate hypothesis of schizophrenia. According to this hypothesis, a relative deficiency of glutamate and consequent hypofunction at the NMDA receptor may contribute to schizophrenia [65]. Early evidence for the hypothesis came from studies involving NMDA receptor antagonists, such as phencyclidine that induced schizophrenia-like positive and negative symptoms in subjects [66]. Studies have shown that activation of the NMDA receptor requires binding of both glutamate and a coagonist Dserine that is a predominant endogenous ligand that binds at the 'glycine' site of the receptor $[67,68]$. Using D-amino oxidase to selectively degrade $\mathrm{D}$-serine is enough to significantly reduce NMDA receptor-mediated neurotransmission [67].

More importantly, D-serine is also a gliotransmitter that is localized to the astrocytes that contain the enzyme serine racemase to convert L-serine to D-serine [69]. Accumulating evidence has demonstrated decreased levels of $\mathrm{D}$-serine in schizophrenia patients, or polymorphisms of the serine racemase gene [70]. In fact, D-serine has proven an efficacious therapeutic agent in the treatment of schizophrenia [70-72]. Recent evidence has put forth a more compelling case for astrocyte function. DISC1 is a gene that was found to be altered in a Scottish family and associated with schizophrenia, depression and bipolar disorder [73]. DISC1 functions in neurodevelopment and regulation of hippocampal adult neurogenesis [73-75]. One recent study has shown how mutant DISC1 may physiologically impair the function of D-serine by binding to serine racemase and causing its degradation and thus resulting in a diminished availability of D-serine at the NMDA receptor [76]. Furthermore, astrocytic DISC1 expression influences adult hippocampal neurogenesis and hippocampal-dependent behaviors through abnormal production of D-serine [77]. Taken together, these studies suggest that astrocyte derived D-serine mediated by DISC1, in addition to NMDA receptor antagonism and reduced glutamate activity may be involved in schizophrenia pathogenesis.

\section{CONCLUSIONS}

Astrocytes play an essential role in the CNS, with roles extending beyond a supportive role as previously thought and into functions such as local network modulation, synaptic plasticity, memory formation, and postinjury repair. Increasing evidence is shedding new light on astrocyte function in major neurological disorders. Future studies will benefit from a focus on astro- 
cyte role in disease etiology as well as exploring possible ways to harness our understanding towards therapeutic benefit.

\section{AUTHOR CONTRIBUTION STATEMENT}

- Full access to all the data in the study and takes responsibility for the integrity of the data and the accuracy of the data analysis: $S M Q H, M H J$

- Study concept and design: $S M Q H, M H J$

- Acquisition of data: $S M Q H$

- Drafting of the manuscript: $S M Q H$

- Critical revision of the manuscript for important intellectual content: $S M Q H$

- Obtained funding: $M H J$

- Administrative, technical, or material support: $M H J$

- Study supervision: $\mathrm{MHJ}$

\section{REFERENCES}

1. Herculano-Houzel S. The glia/neuron ratio: how it varies uniformly across brain structures and species and what that means for brain physiology and evolution. Glia 2014;62:1377-91.

2. Azevedo FA, Carvalho LR, Grinberg LT, Farfel JM, Ferretti RE, Leite RE, et al. Equal numbers of neuronal and nonneuronal cells make the human brain an isometrically scaled-up primate brain. J Comp Neurol 2009;513:532-41.

3. Sherwood CC, Stimpson CD, Raghanti MA, Wildman DE, Uddin M, Grossman LI, et al. Evolution of increased glia-neuron ratios in the human frontal cortex. Proc Natl Acad Sci U S A 2006;103:13606-11.

4. Emsley JG, Macklis JD. Astroglial heterogeneity closely reflects the neuronal-defined anatomy of the adult murine CNS. Neuron Glia Biol 2006;2:175-86.

5. Ota Y, Zanetti AT, Hallock RM. The role of astrocytes in the regulation of synaptic plasticity and memory formation. Neural Plast 2013;2013:185463.

6. Han X, Chen M, Wang F, Windrem M, Wang S, Shanz S, et al. Forebrain engraftment by human glial progenitor cells enhances synaptic plasticity and learning in adult mice. Cell Stem Cell 2013;12:34253.

7. Oberheim NA, Goldman SA, Nedergaard M. Heterogeneity of astrocytic form and function. Methods Mol Biol 2012;814:23-45.

8. Eng LF, Ghirnikar RS, Lee YL. Glial fibrillary acidic protein: GFAPthirty-one years (1969-2000). Neurochem Res 2000;25:1439-51.

9. Goncalves CA, Leite MC, Nardin P. Biological and methodological features of the measurement of $\mathrm{S} 100 \mathrm{~B}$, a putative marker of brain injury. Clin Biochem 2008;41:755-63.

10. Miller RH, Raff MC. Fibrous and protoplasmic astrocytes are biochemically and developmentally distinct. J Neurosci 1984;4:585-92.

11. Cornell-Bell AH, Finkbeiner SM, Cooper MS, Smith SJ. Glutamate induces calcium waves in cultured astrocytes: long-range glial signaling. Science 1990;247:470-3.

12. Charles AC, Merrill JE, Dirksen ER, Sanderson MJ. Intercellular signaling in glial cells: calcium waves and oscillations in response to mechanical stimulation and glutamate. Neuron 1991;6:983-92.

13. Bennett MV, Contreras JE, Bukauskas FF, Saez JC. New roles for astrocytes: gap junction hemichannels have something to communicate. Trends Neurosci 2003;26:610-7.

14. Sofroniew MV, Vinters HV. Astrocytes: biology and pathology. Acta Neuropathol 2010;119:7-35.

15. Volterra A, Meldolesi J. Astrocytes, from brain glue to communication elements: the revolution continues. Nat Rev Neurosci 2005;6:626-40.

16. Haydon PG. GLIA: listening and talking to the synapse. Nat Rev Neurosci 2001;2:185-93.

17. Perea G, Araque A. Properties of synaptically evoked astrocyte calcium signal reveal synaptic information processing by astrocytes. J Neurosci 2005;25:2192-203.

18. Parri HR, Gould TM, Crunelli V. Spontaneous astrocytic $\mathrm{Ca}^{2+}$ oscillations in situ drive NMDAR-mediated neuronal excitation. Nat Neurosci 2001;4:803-12.

19. Nett WJ, Oloff SH, McCarthy KD. Hippocampal astrocytes in situ exhibit calcium oscillations that occur independent of neuronal activity. J Neurophysiol 2002;87:528-37.

20. Haustein MD, Kracun S, Lu XH, Shih T, Jackson-Weaver O, Tong X, et al. Conditions and constraints for astrocyte calcium signaling in the hippocampal mossy fiber pathway. Neuron 2014;82:413-29.

21. Di Castro MA, Chuquet J, Liaudet N, Bhaukaurally K, Santello M, Bouvier D, et al. Local $\mathrm{Ca}^{2+}$ detection and modulation of synaptic release by astrocytes. Nat Neurosci 2011;14:1276-84 .

22. Panatier A, Vallee J, Haber M, Murai KK, Lacaille JC, Robitaille R. Astrocytes are endogenous regulators of basal transmission at central synapses. Cell 2011;146:785-98.

23. Rusakov DA. Disentangling calcium-driven astrocyte physiology. Nat Rev Neurosci 2015;16:226-33.

24. Santello M, Volterra A. Synaptic modulation by astrocytes via $\mathrm{Ca}^{2+}-$ dependent glutamate release. Neuroscience 2009;158:253-9.

25. Bains JS, Oliet SH. Glia: they make your memories stick! Trends Neurosci 2007;30:417-24.

26. Bezzi P, Carmignoto G, Pasti L, Vesce S, Rossi D, Rizzini BL, et al. Prostaglandins stimulate calcium-dependent glutamate release in astrocytes. Nature 1998;391:281-5. 
27. Mothet JP, Pollegioni L, Ouanounou G, Martineau M, Fossier P, Baux G. Glutamate receptor activation triggers a calcium-dependent and SNARE protein-dependent release of the gliotransmitter D-serine. Proc Natl Acad Sci U S A 2005;102:5606-11.

28. Malarkey EB, Parpura V. Mechanisms of glutamate release from astrocytes. Neurochem Int 2008;52:142-54.

29. Luscher C, Malenka RC. NMDA receptor-dependent long-term potentiation and long-term depression (LTP/LTD). Cold Spring Harb Perspect Biol 2012;4.

30. Lisman J, Schulman H, Cline H. The molecular basis of CaMKII function in synaptic and behavioural memory. Nat Rev Neurosci 2002;3:175-90.

31. Isaac JT, Nicoll RA, Malenka RC. Evidence for silent synapses: implications for the expression of LTP. Neuron 1995;15:427-34.

32. Liao D, Hessler NA, Malinow R. Activation of postsynaptically silent synapses during pairing-induced LTP in CA1 region of hippocampal slice. Nature 1995;375:400-4.

33. Anderson CM, Swanson RA. Astrocyte glutamate transport: review of properties, regulation, and physiological functions. Glia 2000;32:114.

34. Bergles DE, Jahr CE. Synaptic activation of glutamate transporters in hippocampal astrocytes. Neuron 1997;19:1297-308.

35. Rusakov DA, Zheng K, Henneberger C. Astrocytes as regulators of synaptic function: a quest for the $\mathrm{Ca}^{2+}$ master key. Neuroscientist 2011;17:513-23.

36. Luscher C, Malenka RC, Nicoll RA. Monitoring glutamate release during LTP with glial transporter currents. Neuron 1998;21:43541.

37. Diamond JS, Bergles DE, Jahr CE. Glutamate release monitored with astrocyte transporter currents during LTP. Neuron 1998;21:425-33.

38. Parpura V, Basarsky TA, Liu F, Jeftinija K, Jeftinija S, Haydon PG. Glutamate-mediated astrocyte-neuron signalling. Nature 1994;369:744-7.

39. Pasti L, Zonta M, Pozzan T, Vicini S, Carmignoto G. Cytosolic calcium oscillations in astrocytes may regulate exocytotic release of glutamate. J Neurosci 2001;21:477-84.

40. Angulo MC, Kozlov AS, Charpak S, Audinat E. Glutamate released from glial cells synchronizes neuronal activity in the hippocampus. J Neurosci 2004;24:6920-7.

41. Tanzi RE. The genetics of Alzheimer disease. Cold Spring Harb Perspect Med 2012;2.

42. Brun A, Liu X, Erikson C. Synapse loss and gliosis in the molecular layer of the cerebral cortex in Alzheimer's disease and in frontal lobe degeneration. Neurodegeneration 1995;4:171-7.

43. Kuchibhotla KV, Lattarulo CR, Hyman BT, Bacskai BJ. Synchronous hyperactivity and intercellular calcium waves in astrocytes in
Alzheimer mice. Science 2009;323:1211-5.

44. Abramov AY, Canevari L, Duchen MR. Changes in intracellular calcium and glutathione in astrocytes as the primary mechanism of amyloid neurotoxicity. J Neurosci 2003;23:5088-95.

45. Yoshiike Y, Kimura T, Yamashita S, Furudate H, Mizoroki T, Murayama $\mathrm{M}$, et al. $\mathrm{GABA}(\mathrm{A})$ receptor-mediated acceleration of aging-associated memory decline in APP/PS1 mice and its pharmacological treatment by picrotoxin. PLoS One 2008;3:e3029.

46. Yoon BE, Jo S, Woo J, Lee JH, Kim T, Kim D, et al. The amount of astrocytic GABA positively correlates with the degree of tonic inhibition in hippocampal CA1 and cerebellum. Mol Brain 2011;4:42.

47. Lee S, Yoon BE, Berglund K, Oh SJ, Park H, Shin HS, et al. Channel-mediated tonic GABA release from glia. Science 2010;330:7906.

48. Jo S, Yarishkin O, Hwang YJ, Chun YE, Park M, Woo DH, et al. GABA from reactive astrocytes impairs memory in mouse models of Alzheimer's disease. Nat Med 2014;20:886-96.

49. Kessler RC, Berglund P, Demler O, Jin R, Koretz D, Merikangas KR, et al. The epidemiology of major depressive disorder: results from the National Comorbidity Survey Replication (NCS-R). JAMA 2003; 289:3095-105.

50. Ongur D, Drevets WC, Price JL. Glial reduction in the subgenual prefrontal cortex in mood disorders. Proc Natl Acad Sci U S A 1998; 95:13290-5.

51. Cotter D, Mackay D, Landau S, Kerwin R, Everall I. Reduced glial cell density and neuronal size in the anterior cingulate cortex in major depressive disorder. Arch Gen Psychiatry 2001;58:545-53.

52. Banasr M, Duman RS. Glial loss in the prefrontal cortex is sufficient to induce depressive-like behaviors. Biol Psychiatry 2008;64: 863-70.

53. Bremner JD, Narayan M, Anderson ER, Staib LH, Miller HL, Charney DS. Hippocampal volume reduction in major depression. Am J Psychiatry 2000;157:115-8.

54. Stockmeier CA, Mahajan GJ, Konick LC, Overholser JC, Jurjus GJ, Meltzer HY, et al. Cellular changes in the postmortem hippocampus in major depression. Biol Psychiatry 2004;56:640-50.

55. Lima A, Sardinha VM, Oliveira AF, Reis M, Mota C, Silva MA, et al. Astrocyte pathology in the prefrontal cortex impairs the cognitive function of rats. Mol Psychiatry 2014;19:834-41.

56. Cao X, Li LP, Wang Q, Wu Q, Hu HH, Zhang M, et al. Astrocytederived ATP modulates depressive-like behaviors. Nat Med 2013; 19:773-7.

57. Klempan TA, Sequeira A, Canetti L, Lalovic A, Ernst C, ffrenchMullen J, et al. Altered expression of genes involved in ATP biosynthesis and GABAergic neurotransmission in the ventral prefrontal 
cortex of suicides with and without major depression. Mol Psychiatry 2009;14:175-89.

58. Ortinski PI, Dong J, Mungenast A, Yue C, Takano H, Watson DJ, et al. Selective induction of astrocytic gliosis generates deficits in neuronal inhibition. Nat Neurosci 2010;13:584-91.

59. Fedele DE, Gouder N, Guttinger M, Gabernet L, Scheurer L, Rulicke $\mathrm{T}$, et al. Astrogliosis in epilepsy leads to overexpression of adenosine kinase, resulting in seizure aggravation. Brain 2005;128:2383-95.

60. Manning TJ Jr, Sontheimer H. Spontaneous intracellular calcium oscillations in cortical astrocytes from a patient with intractable childhood epilepsy (Rasmussen's encephalitis). Glia 1997;21:332-7.

61. Lee SH, Magge S, Spencer DD, Sontheimer H, Cornell-Bell AH. Human epileptic astrocytes exhibit increased gap junction coupling. Glia 1995;15:195-202.

62. Fellin T, Gomez-Gonzalo M, Gobbo S, Carmignoto G, Haydon PG. Astrocytic glutamate is not necessary for the generation of epileptiform neuronal activity in hippocampal slices. J Neurosci 2006; 26:9312-22.

63. Gomez-Gonzalo M, Losi G, Chiavegato A, Zonta M, Cammarota $\mathrm{M}$, Brondi $\mathrm{M}$, et al. An excitatory loop with astrocytes contributes to drive neurons to seizure threshold. PLoS Biol 2010;8:e1000352.

64. Ding S, Fellin T, Zhu Y, Lee SY, Auberson YP, Meaney DF, et al. Enhanced astrocytic $\mathrm{Ca}^{2+}$ signals contribute to neuronal excitotoxicity after status epilepticus. J Neurosci 2007;27:10674-84.

65. Tsai G, Coyle JT. Glutamatergic mechanisms in schizophrenia. Annu Rev Pharmacol Toxicol 2002;42:165-79.

66. Jentsch JD, Roth RH. The neuropsychopharmacology of phencyclidine: from NMDA receptor hypofunction to the dopamine hypothesis of schizophrenia. Neuropsychopharmacology 1999;20:201-25.

67. Mothet JP, Parent AT, Wolosker H, Brady RO Jr, Linden DJ, Ferris $\mathrm{CD}$, et al. D-serine is an endogenous ligand for the glycine site of the N-methyl-D-aspartate receptor. Proc Natl Acad Sci U S A 2000; 97:4926-31.
68. Panatier A, Theodosis DT, Mothet JP, Touquet B, Pollegioni L, Poulain DA, et al. Glia-derived D-serine controls NMDA receptor activity and synaptic memory. Cell 2006;125:775-84.

69. Wolosker H, Blackshaw S, Snyder SH. Serine racemase: a glial enzyme synthesizing D-serine to regulate glutamate-N-methyl-D-aspartate neurotransmission. Proc Natl Acad Sci U S A 1999;96:1340914.

70. Labrie V, Wong AH, Roder JC. Contributions of the D-serine pathway to schizophrenia. Neuropharmacology 2012;62:1484-503.

71. Tsai G, Yang P, Chung LC, Lange N, Coyle JT. D-serine added to antipsychotics for the treatment of schizophrenia. Biol Psychiatry 1998;44:1081-9.

72. Kantrowitz JT, Malhotra AK, Cornblatt B, Silipo G, Balla A, Suckow RF, et al. High dose D-serine in the treatment of schizophrenia. Schizophr Res 2010;121:125-30.

73. Millar JK, Wilson-Annan JC, Anderson S, Christie S, Taylor MS, Semple CA, et al. Disruption of two novel genes by a translocation co-segregating with schizophrenia. Hum Mol Genet 2000;9:141523.

74. Duan X, Chang JH, Ge S, Faulkner RL, Kim JY, Kitabatake Y, et al. Disrupted-In-Schizophrenia 1 regulates integration of newly generated neurons in the adult brain. Cell 2007;130:1146-58.

75. Faulkner RL, Jang MH, Liu XB, Duan X, Sailor KA, Kim JY, et al. Development of hippocampal mossy fiber synaptic outputs by new neurons in the adult brain. Proc Natl Acad Sci U S A 2008;105:14157-62.

76. Ma TM, Abazyan S, Abazyan B, Nomura J, Yang C, Seshadri S, et al. Pathogenic disruption of DISC1-serine racemase binding elicits schizophrenia-like behavior via D-serine depletion. Mol Psychiatry 2013;18:557-67.

77. Terrillion CE, Abazyan B, Yang Z, Crawford J, Shevelkin AV, Jouroukhin Y, et al. DISC1 in astrocytes influences adult neurogenesis and hippocampus-dependent behaviors in mice. Neuropsychopharmacology 2017;42:2242-51. 